\title{
Multifocal Electroretinogram in normal subjects
}

\author{
Ahmad Mostafa Ahmad Abdallah ${ }^{1}$; MD, Hatem Gamal Ammar ${ }^{1}$; MD, Kariman \\ GTA $^{1}$; BBCH, Yoshiaki Shimada ${ }^{2}$; MD \\ 1 Ophthalmology Department, Sohag Faculty of Medicine, Sohag University, Egypt. \\ 2 Ophthalmology Department, Fujita Health University School of Medicine, Nagoya, \\ Japan. \\ Corresponding author; Kariman GTA \\ Ophthalmology Department, Sohag Faculty of Medicine, Sohag University, Egypt.
}

\begin{abstract}
Background: Multifocal electroretinogram (mfERG) is a device for recording the physiological response of multiple retinal areas in short time. Using this technique enables us to detect the spatial extent and severity of the retinal damage. Objectives: In this study,we aimed to assess the topographic properties of mfERG in normal subjects to use it as a basic data to evaluate eye disease. Methods: We recorded mfERG from 20 eyes of $\mathbf{2 0}$ normal subjects. We measured amplitude and latency of recorded waves. We compared between amplitude of these waves between different retinal areas and the same for latency. Results: We found mfERG responses in the form of waves, every wave consists of negative wave (N1) followed by positive wave (P1). The amplitude of N1 wave and amplitude of P1 were largest at the fovea and decreased toward periphery. The latency of N1 and P1 were shorter in upper retina than lower retina. The N1 amplitude and P1 amplitude was larger in upper retina than lower retina, which reflex functional superiority of upper retina. There was no statistical difference between nasal and temporal retina in the amplitude and latency.Conclusion: Multi-focal ERG would be useful for objective examination of the retinal function.
\end{abstract}

Key words:mfERG; retina; topography; functional superiority of upper retina.

\section{Introduction}

The multifocal electroretinogram (mfERG) is a relatively new technique for assessing the local ERG from different regions of the posterior retina. Itwas developed by Sutter in 1992, which measures the spatial distribution of the central retinal cone function (1). The mfERG extracts many local responses simultaneously by mathematical analysis through pseudorandom light stimuli following binary $m$-sequence. The result of mfERG is obtained from multiple retinal areas simultaneously in short time and the amplitude of response is reported to correspond to the density of the cones (2). Electrical responses from the eye are recorded with a corneal electrode just as in conventional ERG recording, but the special nature of the stimulus and analysis produce a topographic map of ERG responses (3).mfERG is a very sensitive tool in detection of localized retinal dysfunction in a patient who had normal full-field ERG (4). In our study, we aimed to analyze the topographical properties of mfERG responses in multiple retinal areas in normal eyesto use it as a basic data to evaluate eye with pathology.

\section{Subjects and Methods:}

This study was applied to healthy subjects (volunteers) from TOMEY corporation company, Nagoya, Japan. Informed consent was obtained from 
each subject after a full explanation of the procedures. This study was approved by the ethical committee review board of Fujita Health University, Nagoya, Japan and Sohag University, Egypt. Multifocal ERGs were recorded in $\mathbf{2 0}$ eyes of $\mathbf{2 0}$ normal subjects with no ocular disease except for refractive error. The eye of the subject's choice was tested (11 right eyes and $\mathbf{9}$ left). Subjects ranging in age from 31-53 years (mean=42.35 years). All subjects received eye examination including a fundus examination with dilated pupils. The visual acuity was $\mathbf{1 . 0}$ or better with correction. Refractive error ranged from $\mathbf{0}$ to $\mathbf{- 6}$ diopters. The mean spherical equivalent refractive error of these subjects was $\mathbf{- 2 . 9 4}$ Diopters $( \pm \mathbf{1 . 9 2} \mathrm{D})$. All subjects were free of ocular and systemic pathology. Recording:

All subjects were subjected to full ophthalmological examination including visual acuity, slit-lamp examination, intraocular pressure, and direct and indirect ophthalmoscope. The recordings were performed under room light conditions.

Pupils were dilated with eye drops of $\mathbf{0 . 5 \%}$ tropicamide and $0.5 \%$ phenylephrine hydrochloride. Subjects sit comfortably in front of the screen. The pupil was centered in the corneal electrode ring using contact lens electrodes. Two drops of topical anesthetic were placed on the eye prior to inserting the contact lens electrode. Celluvisc (Allergan, Irvine, CA) was used to protect the cornea under the contact lens electrode. If more than two blinks were present in a $\mathbf{3 0}$ sec segment, the data for that segment were discarded and the segment repeated. A ground electrode was clipped to the left ear lobe.Good fixation, both central and steady, was done to avoid eccentric fixation. Refraction was corrected by using lenses that was placed in a holder in front of the eye.

Response analysis:

The waveforms in mfERG consist of negative peak called (N1) wave and positive peak called (P1) wave.The amplitude of the N1-wave was measured from the baseline to the bottom of the N1-wave, and the amplitude of P1-wave was from the trough of the N1-wave to the peak of the P1wave. The peak latency was defined as the period from the time stimulation was given to the peak of each wave (Figure 1).

Responses from all elements of the stimulus were grouped to learn the topographical properties of retinal responses as follows: (a) fovea and its outer portions, (b) upper and lower retina, and (c) nasal and temporal retina.The amplitude (nv/deg ${ }^{2}$ ) and the latency (milliseconds) of the first negative wave (N1A and N1L, respectively), and the first positive wave amplitude and latency (P1A and P1L, respectively) were recorded. The data obtained were exported to excel sheet. Statistical analysis was carried out using the excel 2013. Analysis of variance (ANOVA) was used to correlate the change in mfERG responses with increasing eccentricity from the fovea, paired $\mathrm{t}$-tests for the upper and lower retina, and the nasal and temporal retina. $\boldsymbol{P}<\mathbf{0 . 0 5}$ was considered statistically significant. 


\section{Results}

\section{Comparison between the Fovea and Its Outer Portions:}

We grouped responses in a trace array into four areas and named the areas $\mathbf{1}$ to $\mathbf{4}$, respectively, from the center to the periphery (Figure 2). Responses were summed and averaged in each area. A negative wave (N1-wave) and a positive wave (P1-wave) were found in all areas. Statistical difference was found, but there was a tendency for N1 latency to be long at the fovea, shorter at the parafovea, and again longer at the perifovea $(\mathrm{P}=\mathbf{0 . 0 1})$ (Table 1, Figure 3A). Also The $\mathrm{P} 1$ latency was long at the fovea, shorter at the parafovea, and again longer at the perifovea $(\mathrm{P}=\mathbf{0 . 5})$ (Figure 3C). The N1 and $\mathrm{P} 1$ amplitudes decreased from the fovea outward $(\mathrm{P}<\mathbf{0 . 0 0 0 1})$ (Figures $\mathbf{3}$ ).

\section{Comparison between the Upper and Lower Retina}

Each wave grouped in the upper and lower retina as shown in (Fig.4) and summed and averaged focal ERGs in each group. The latency of the N1 wave was statistically shorter in the upper retina than in the lower retina (Table $\mathbf{2}, \mathbf{P}<\mathbf{0 . 0 0 0 1}$ ). The $\mathrm{N} 1$ amplitude was statistically larger in the upper than in the lower retina (Table $\mathbf{2}, \mathrm{P}=\mathbf{0 . 0 0 0 8}$ ). The $\mathrm{P} 1$ amplitude was statistically larger in the upper than in the lower retina (Table 2, $\mathrm{P}=$ 0.0001). The latency of the $P 1$ wave was statistically shorter in the upper retina than in the lower retina (Table $\mathbf{2}, \mathrm{P}<\mathbf{0 . 0 0 0 1}$ ).

\section{Comparison between the Temporal and Nasal Retina}

We grouped each wave in the nasal and temporal retina as shown in (Figure 5) and totaled and averaged focal ERGs in each group. The portion including the optic disc was excluded from the summation. No statistical difference was found in N1 latency (P $=\mathbf{0 . 0 4})$, N1 amplitude $(P=\mathbf{0 . 3 2})$, $P 1$ latency $(P=\mathbf{0 . 1 3})$, or $P 1$ amplitude $(P=\mathbf{0 . 1 2})$ as shown in (Table 3).

\section{Discussion}

ERG response amplitudes are widely used to quantify retinal activity (5). In this study, the amplitudes of N1-waves and P1-waves were the largest in the fovea, and they decreased toward periphery.Song et al. (6)reported thatwith the increasing of eccentricity, $\mathrm{P} \mathbf{1}$ amplitude (all $\mathrm{p}=\mathbf{0 . 0 0 0 0}$ in ring $\mathbf{1}$-5) of each group decreased.Azad et al. (7) reported that the amplitudes of the N1 and P1 waves also were largest in the fovea (area 1) and decreased progressively with increasing eccentricity. This observation may be explained anatomically by the cone density distribution, as demonstrated by Curcio et al. (8) from cadaveric retinas. Sutter and Tran (2) reported before thatthe multifocal ERG responses fell with eccentricity. Nagatomo et al (9) recorded multifocal ERGs from 20 eyes

of 20 normal subjects and analyzed the topographical properties of the responses and reported that the mean amplitudes were largest in foveal area and decreased with eccentricity by using a 61 element stimulus pattern. Hood et al (10) reported that the latencies can be a sensitive index of retinaldamage in some eye disease. In our study, the latencies (Implicit Times) of N1-waves tended to be long in the fovea, become shorter in the parafovea, and again longer in the periphery. Also Implicit times for P1-waves show a slight tendency for foveal responses to be longer than parafoveal. Nagatomo et al. (9) reported the same findings.But the underlying physiologic mechanism remains unknown.

In contrast, Azad et al. (11) reported that the latencies of the P1 and $\mathbf{N} 2$ 
SOHAG MEDICAL JOURNAL

Vol. 21 No.1 Jan 2017
Multifocal Electroretinogram in normal subjects Kariman Gamal Tamam waves were longest in the central ring and progressively shortened with eccentricity. A similar finding was however not obtained in the latencies of the N1 wave.

It is noticed that the N1 and P1 amplitude were larger in the upper retina than in the lower retina. The N1 and P1 latencies were shorter in the upper retina than in the lower retina. Also Nagatomo et al. (9) have reported shorter latencies and larger amplitudes of upper over lower retina in the average of $\mathbf{2 0}$ eyes.A functional asymmetry between the upper retina and lower retina has been reported by visual acuity, and the standing potential of the eye (12). These reports suggest a superior visual function in the upper retina over the lower retina. This proved that the new technique of multifocal ERG indeed reflects the local function of the outer retina.

No statistical difference was found between latency and amplitude of the nasal and temporal retina. But Sutter and Tran (2) reported that there was asymmetry between the nasal and temporal areas in every subject and the nasal retina showed higher response density. Nagatomo et al (9) and Wane et al (5) reported no statistical difference between the nasal and temporal areas which is the same in the present study.

\section{Figures:}

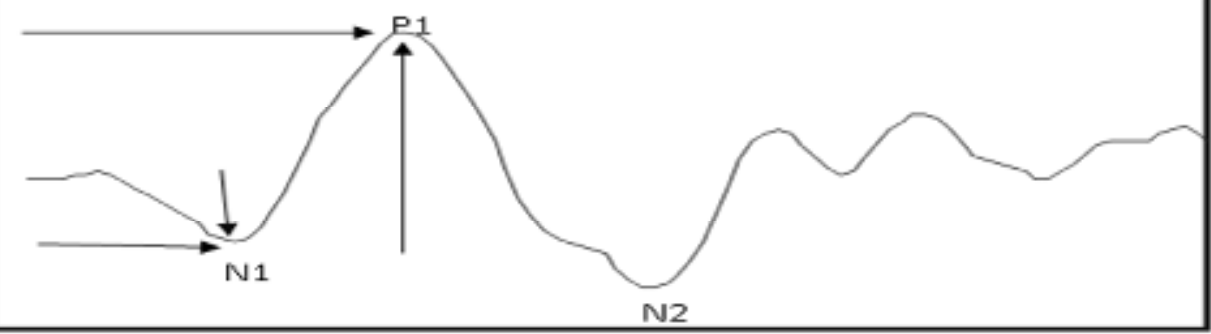

Fig. 1: Diagram of mfERG response to show the designation of the major features of the waveform. The arrows show the trough-to-peak amplitude (vertical arrow) and the implicit time (horizontal arrow) from the onset of the stimulus to the peak of the waveform.

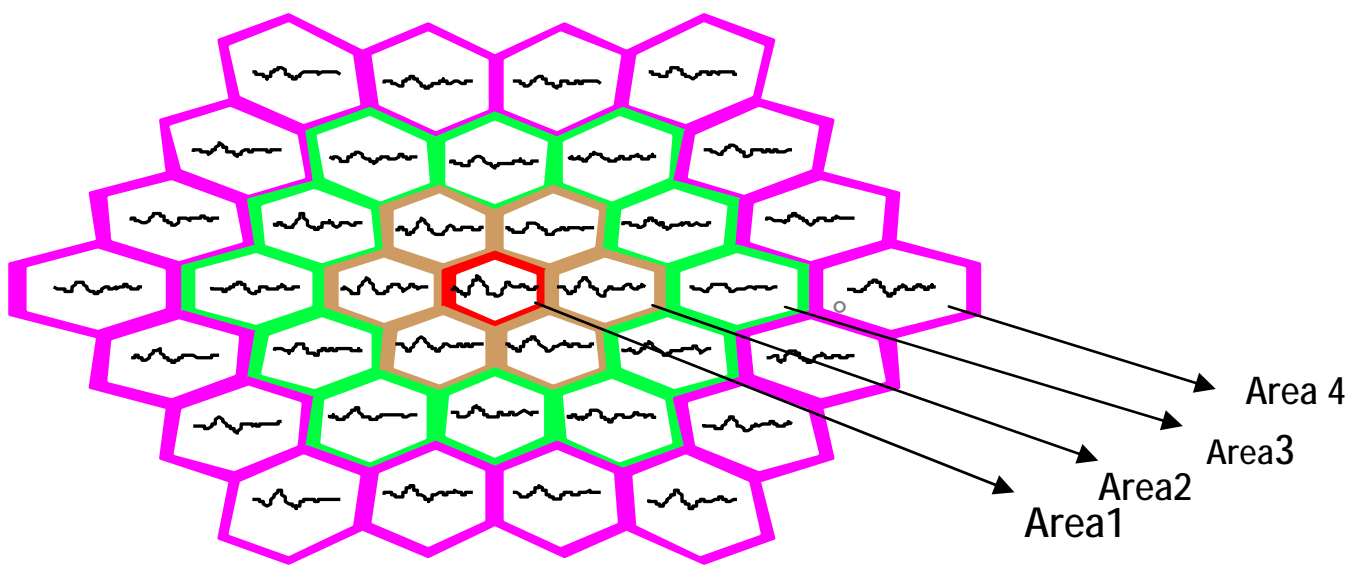

Fig. 2: Comparison between fovea and its outer portions. Area1 corresponds to fovea, area 2 to parafovea, areas $\mathbf{3}$ and area $\mathbf{4}$ to the outer portions. 


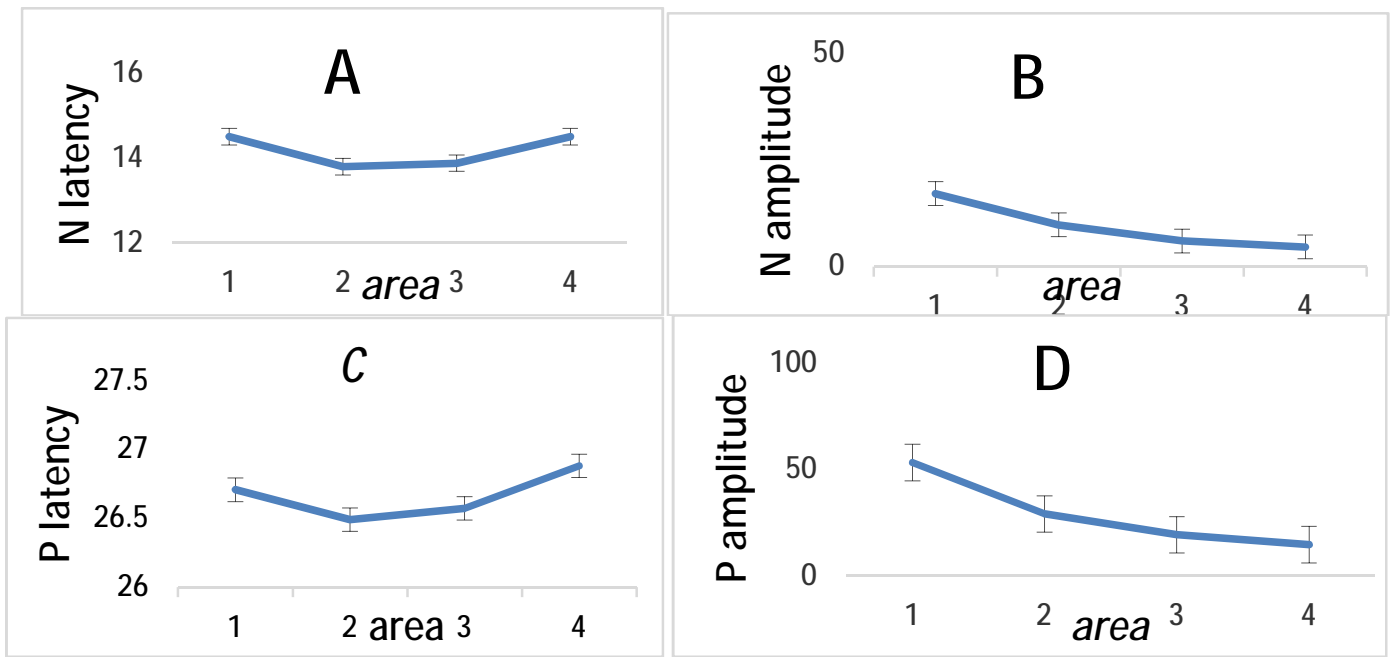

Fig.3. Comparison between fovea and its outer portions. Mean and standard error of N1 latency (A), N1 amplitude (B), P1 latency (C), and P1 amplitude (D) at four different stimulus sites illustrated in Figure $\mathbf{2}$.

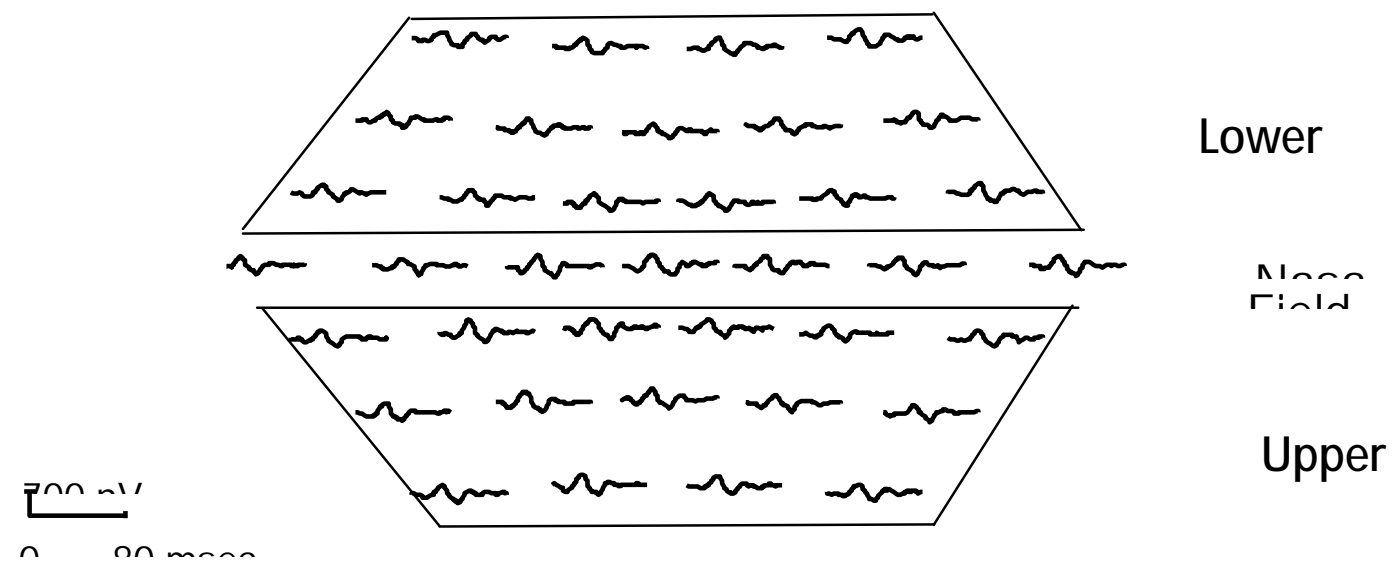

Fig.4. Comparison between the upper retina (lower half array) and lower retina (upper half array).

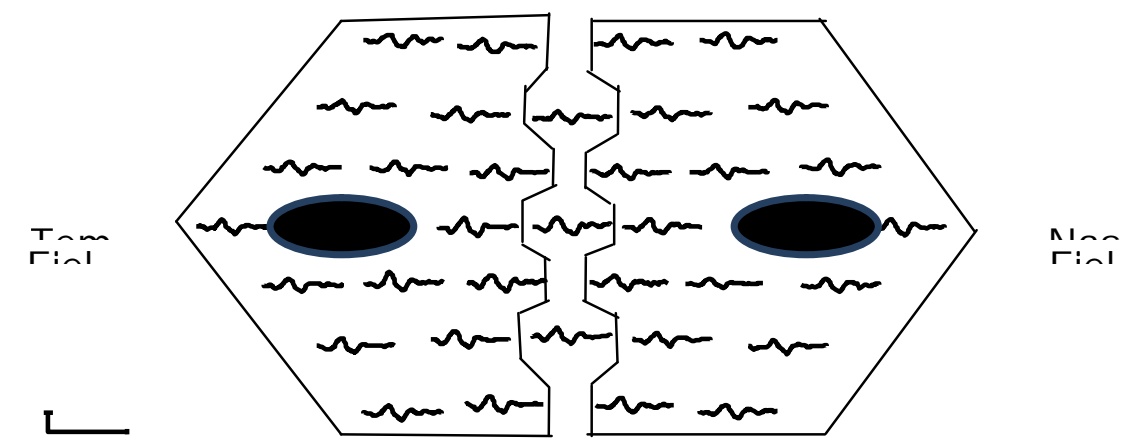


Fig.5. Comparison between the nasal and temporal retina, the portion that included the optic disc and the nasal portion corresponding to this black circular portion was excluded from the summation.

Tables

Table 1. Latency (msec) and Amplitude $\left(\mathrm{nV} / \mathrm{deg}^{2}\right)(\mathrm{Mean} \pm$ Standard Error) of the N1 and P1-Waves at Four Different Stimulus Sites Illustrated in Figure 2.

\begin{tabular}{|r|r|r|r|r|}
\hline & area1 & area2 & area3 & area4 \\
\hline N1 Amplitude & $17.13 \pm 1.58$ & $9.80 \pm 0.69$ & $\mathbf{6 . 0 3} \pm 0.44$ & $4.61 \pm 0.34$ \\
\hline N1Latency & $14.50 \pm 0.28$ & $13.79 \pm 0.18$ & $13.87 \pm 0.18$ & $14.50 \pm 0.17$ \\
\hline P1 Amplitude & $53.28 \pm 4.64$ & $29.19 \pm 21.99$ & $19.39 \pm 1.32$ & $14.82 \pm 0.93$ \\
\hline P1 Latency & $26.70 \pm 0.20$ & $26.49 \pm 0.22$ & $26.57 \pm 0.24$ & $26.88 \pm 0.23$ \\
\hline
\end{tabular}

Table 2. Latency (msec) and Amplitude (nV) (Mean \pm Standard Error) of the $\mathrm{N}$ - and P1-Waves at the Upper and Lower Retina.

\begin{tabular}{|r|r|r|}
\hline & Upper Retina & lower Retina \\
\hline N1 Amplitude & $\mathbf{6 . 1 3} \pm 0.41$ & $\mathbf{5 . 2 4} \pm 0.44$ \\
\hline N1 Latency & $13.58 \pm 0.16$ & $14.98 \pm 0.14$ \\
\hline P1 Amplitude & $18.67 \pm 1.17$ & $17.04 \pm 1.10$ \\
\hline P1 Latency & $25.66 \pm 0.80$ & $\mathbf{2 6 . 7 0} \pm 0.76$ \\
\hline
\end{tabular}

Table 3. Latency (msec) and Amplitude (nV) (Mean \pm Standard Error) of the N-and P1-Waves at the Temporal and Nasal Retina.

\begin{tabular}{|l|r|r|}
\hline & temporal Retina & Nasal Retina \\
\hline N1Amplitude & $\mathbf{5 . 6 2} \pm 0.42$ & $5.85 \pm 0.41$ \\
\hline N1 Latency & $14.15 \pm 0.16$ & $14.41 \pm 0.15$ \\
\hline P1Amplitude & $17.45 \pm 1.45$ & $18.40 \pm 1.13$ \\
\hline P1 Latency & $26.37 \pm 0.74$ & $26.16 \pm 0.20$ \\
\hline
\end{tabular}




\section{References}

1- Hood DC. Assessing retinal with the multifocal technique. Prog Retin Eye

Res.2000 ; 19 : 607-646.

2- Sutter EE, Tran D.the field topography of ERG components in man 1.The p3Usui S, Nagasaka E. Spatial distribution of local flash electroretinogram by multi-input stimulation. Doc ophthalmic.1994; 88 : 57-63.

4-Timothy Y.Y. Lai, MMedSc, MRCSEd, Wai-Man Chan, MRCP, FRCSEd, Ricky Y.K. Lai, MMedSc, Jasmine W.S. Ngai, MRCSEd, Haitao Li, MD, and Dennis S.C. Lam, MD, FRCOphth. The Clinical Applications of Multifocal Electroretinography: A Systematic Review. Surv Ophthalmol .2007; 52:6196.hotonic luminance response. Vision Res. 1992 ; 32 : 433-446.

5- WAYNE A. VERDON \& GUNILLA HAEGERSTROM-PORTNOY.

Topography of the multifocal electroretinogram. Documenta Ophthalmologica. 1998; 95: 73-90.

6- Song AP, Yu T, Wang JR, Liu W, Sun Y, Ma SX. Multifocal electroretinogram in non-pathological myopic subjects: correlation with optical coherence tomography. Intel ophthalmol. 2016;9(2):286-291.
7- Rajvardhan Azad, Urmimala Ghatak, Yog Raj Sharma, and Parijat Chandra. Multifocal electroretinogram in normal Emmetropic subjects. Indian $\mathrm{J}$ Ophthalmol. 2012 Jan-Feb; 60(1): 4952.

8- Curcio CA, Sloan KR Jr, Packer O, Hendrickson AE, Kalina RE. Distribution of cones in human and monkey retina: Individual variability and radial asymmetry. Science. 1987; 236:579-82.

9- Akiko Nagatomo, Nobuhisa Nao-i, Futoshi Maruiwa, Mikki Arai, Atsushi Sawada. Multifocal Electroretinograms in Normal Subjects. Jpn J Ophthalmol.1998; 42:129-135.

10-Hood DC, Holopigian K, Greenstein V, et al. Assessment of local retinal function in patients with retinitis pigmentosa using the multi-focal ERG technique. Vision Research .1998; 38(1): 163-79.

11-Rajvardhan Azad, Urmimala Ghatak, Yog Raj Sharma, and Parijat Chandra. Multifocal electroretinogram in normal Emmetropic subjects. Indian $\mathrm{J}$ Ophthalmol. 2012 Jan-Feb; 60(1): 4952.

12- Skrandies W, Baier M. The standing potential of the human eye reflects differences between upper and lower retinal areas. Vision Res. 1986; 26:57781. 